\title{
Nine Seasons of a Bronchiolitis Observation Unit and Home Oxygen Therapy Protocol
}

\author{
Timothy J D Ohlsen, MD ${ }^{1 *}$, Alexander M Knudson, MPH², E Kent Korgenski, MT, MS, \\ David R Sandweiss, MD³, Michelle G Hofmann, MD, MPH${ }^{4}$, Tiffany S Glasgow, MD¹, Eric R Coon, MD, MS
}

\begin{abstract}
${ }^{1}$ Department of Pediatrics, Division of Inpatient Medicine, University of Utah, Salt Lake City, Utah; ${ }^{2}$ University of Utah School of Medicine, Salt Lake City, Utah; ${ }^{3}$ Department of Pediatrics, Division of Emergency Medicine, University of Utah, Salt Lake City, Utah; ${ }^{4}$ Department of Pediatrics, Division of General Pediatrics, Salt Lake City, Utah.
\end{abstract}

BACKGROUND: We implemented an observation unit and home oxygen therapy (OU-HOT) protocol at our children's hospital during the 2010-2011 winter season to facilitate earlier discharge of children hospitalized with bronchiolitis. An earlier study demonstrated substantial reductions in inpatient length of stay and costs in the first year after implementation.

OBJECTIVE: Evaluate long-term reductions in length of stay and cost.

DESIGN, SETTING, AND PARTICIPANTS: Interrupted time-series analysis, adjusting for patient demographic factors and disease severity. Participants were children aged 3 to 24 months and hospitalized with bronchiolitis from 2007 to 2019.

INTERVENTION: OU-HOT protocol implementation.

MAIN OUTCOME AND MEASURES: Hospital length of stay. Process measures were the percentage of patients discharged from the OU; percentage of patients discharged with HOT. Balancing measures were 7-day hospital revisit rates; annual per-population bronchiolitis admission rates. Secondary outcomes were inflation-adjusted cost per episode of care and discharges within 24 hours.

RESULTS: A total of 7,116 patients met inclusion criteria. The OU-HOT protocol was associated with immediate decreases in mean length of stay ( -30.6 hours; $95 \%$ $\mathrm{Cl},-37.1$ to -24.2 hours) and mean cost per episode of care $(-\$ 4,181 ; 95 \% \mathrm{Cl},-\$ 4,829$ to $-\$ 3,533)$. These findings were sustained for 9 years after implementation. Hospital revisit rates did not increase immediately $(-1.1 \%$ immediate change; $95 \% \mathrm{Cl},-1.8 \%$ to $-0.4 \%)$, but a small increase in revisits was observed over time (change in slope $0.4 \%$ per season, $95 \% \mathrm{Cl}, 0.1 \%-0.8 \%$ ).

CONCLUSION: The OU-HOT protocol was associated with sustained reductions in length of stay and cost, representing a promising strategy to reduce the inpatient burden of bronchiolitis. Journal of Hospital Medicine 2021;16:261-266. (C) 2021 Society of Hospital Medicine ronchiolitis is the leading cause of hospitalization in infants aged $<1$ year in the United States. ${ }^{1-3}$ Estimates suggest that $1.5 \%$ to $2.0 \%$ of US infants require hospitalization every year, with a median (interquartile range) length of stay of 2 days $(1-4),{ }^{3}$ incurring direct medical costs of $\$ 555$ million annually. ${ }^{1}$ Evidence suggests that few interventions, aside from supportive care, are effective for bronchiolitis. ${ }^{4-7}$ Adherence to standardized clinical guidelines could improve outcomes and resource use by streamlining care and limiting ineffective interventions, thereby decreasing hospital length of stay, which is a major medical cost. ${ }^{8-13}$ For this reason, many hospitals have adopted bronchiolitis guidelines, although institutional practices vary. ${ }^{14,15}$

Two relatively unexplored methods to reduce the inpatient burden of bronchiolitis are the use of observation units (OU)

Corresponding Author: Timothy J D Ohlsen, MD;

Email: timothy.ohlsen@seattlechildrens.org. Twitter: @TimOhlsenMD.

Published online first April 20, 2021

Received: May 23, 2020; Revised: November 2, 2020;

Accepted: November 22, 2020

(C) 2021 Society of Hospital Medicine DOI 10.12788/jhm.3576 and home oxygen therapy (HOT). Motivated by research demonstrating the safety and effectiveness of an emergency department (ED)-based HOT protocol, ${ }^{16}$ where 36 of 37 patients with mild hypoxemia discharged on HOT avoided hospital admission, our institution implemented an observation unit and home oxygen therapy (OU-HOT) protocol designed to return children with bronchiolitis home earlier from the hospital. In the first winter season of implementation (2010 to 2011), the OU-HOT protocol was associated with significant reductions in length of stay and substantial cost savings, without an increase in return visits to the ED or inpatient readmissions. ${ }^{17}$ The objectives of this study were to determine whether these encouraging initial findings persisted and to measure the long-term impact of the OU-HOT protocol.

\section{METHODS}

We conducted a retrospective cohort study of children hospitalized with bronchiolitis at Primary Children's Hospital, a freestanding children's hospital in Salt Lake City, Utah. Discharge diagnosis and procedures codes, as well as laboratory, imaging, pharmacy, and supply costs, were obtained from the Intermountain Healthcare enterprise data warehouse. A crosswalk 
available from the Centers for Medicare and Medicaid Services was used to convert International Classification of Diseases (ICD)-10 discharge diagnosis and procedure codes to ICD-9 equivalents. ${ }^{18}$ This study was approved by the University of Utah institutional review board (00110419).

\section{Patients}

Children aged 3 to 24 months who were discharged with a diagnosis of bronchiolitis (466.xx) during winter seasons from 2007 to 2019 were included. A winter season was defined as November 1 to April 30. Both observation and inpatient encounters were included in the cohort. We excluded patients with discharge diagnosis or procedure codes indicating tracheostomy (519.0-519.09, V44.0, V55.0, 31.1, 31.21, 31.41, 31.74, 97.23), ventilator dependence (V46.1x), chronic lung disease (518.83, 770.7), or pulmonary hypertension (416.xx). Patients with both bronchiolitis and a concurrent diagnosis, such as otitis media or pneumonia, were included unless exclusion criteria were met.

\section{Intervention and Process Measures}

Our institution implemented the OU-HOT protocol at the start of the 2010-2011 winter season. ${ }^{17}$ The aim of the OU-HOT protocol was to discharge children with bronchiolitis home sooner by increasing use of both an $\mathrm{OU}$, with frequent assessment of discharge readiness, and HOT to help children become ready for discharge. Similar to most OUs, admission to our unit was limited to patients who met hospital admission criteria, and had a short anticipated length of stay ( $<48$ hours). As a self-contained 20-bed unit providing 24-hour dedicated pediatrician/ pediatric emergency medicine physician and nursing coverage, the $\mathrm{OU}$ actively monitored patients' discharge readiness, with a goal to facilitate patient throughput more akin to an ED rather than a traditional inpatient unit. Patients who could not be discharged from the $\mathrm{OU}$ within 48 hours were transferred to the inpatient unit. Although the $\mathrm{OU}$ existed at the time of protocol implementation, its use for patients with bronchiolitis was not actively encouraged until implementation.

Hospitalized patients - in either inpatient or observation units -were eligible for discharge on HOT if they met the following criteria: hypoxemia was the only indication for continued hospitalization, the child's oxygen requirement was $<0.5 \mathrm{~L} / \mathrm{min}$ for at least 6 hours $(0.8 \mathrm{~L} / \mathrm{min}$ for children aged $>1$ year), the child's caregiver(s) were willing to manage oxygen at home, and the child had reliable access to primary care provider follow up. We used two process measures across winter seasons: (1) the percentage of patients discharged from the $\mathrm{OU}$, and (2) the percentage of patients discharged with HOT. The percentage of patients discharged on HOT was estimated by a manual chart review and an electronic medical record (EMR) HOT flag that came into existence with our hospital system's adoption of a new EMR (2017-2019). Chart review randomly sampled patients from 2007-2017, totaling 457 patients. To estimate the reliability of this method, we calculated the sensitivity, specificity, positive predictive value, and negative predictive value of the EMR HOT flag using chart review as the gold standard.

\section{Outcome Measures}

The main outcome measure was mean hospital length of stay. Balancing measures were revisit rates (stratified into ED visits and readmissions) and annual per-population bronchiolitis admission rates. Visits were considered revisits if they occurred within 7 days of initial hospital discharge, and included visits to Primary Children's Hospital as well as 22 other Intermountain Healthcare hospitals. Population estimates from the Utah Department of Health were used to calculate the annual population-based rate of bronchiolitis admissions to Primary Children's Hospital. ${ }^{19}$ Annual admission rates were calculated per 10,000 children aged 3 to 24 months who resided in Utah each year of the study period, and were evaluated to determine if patients were admitted more frequently after OU-HOT implementation. Secondary outcome measures included the percentage of patients discharged within 24 hours and mean inflation-adjusted cost per episode of care (in 2019 dollars). Hospitalization costs were determined using Intermountain Healthcare's internal cost accounting system, an activity-based method that aggregates costs of individual resources according to date of service. ${ }^{20}$ Costs were adjusted to 2019 dollars and were defined as the total costs of a patient's initial hospitalization as well as any 7-day revisit encounters.

\section{Data Analysis}

Demographic data were compared before and after OU-HOT protocol implementation using Pearson chi-square tests. Multivariable linear or logistic regression models were used to compare measures before and after OU-HOT protocol implementation via an interrupted time-series approach. The interrupted time-series analysis measured two types of changes after protocol implementation during the 2010-2011 winter season: (1) any immediate change in the level of an outcome (immediate effect) and (2) any change of an outcome going forward over time (change in slope). ${ }^{21}$ Covariates in the regression models included patient age, sex, race, ethnicity, and insurance type, as well as presence of an underlying complex chronic condition, mechanical ventilation use, and pediatric intensive care unit (PICU) admission during hospitalization. Data were analyzed in STATA 15 (StataCorp LLC). ${ }^{22}$

\section{RESULTS}

A total of 7,116 patients met inclusion criteria over the study period (2,061 pre-implementation, 5,055 post-implementation). A comparison of patient characteristics before and after HOT protocol implementation is presented in Table 1. Patients were similar in terms of age, sex, and insurance type. Patients in the postimplementation period were more likely to have a complex chronic condition, require admission to the PICU, and need mechanical ventilation $(P<.01)$. Differences between cohorts with regard to race/ethnicity distribution largely were a result of improved capture of these data elements in the postimplementation period. For example, $30 \%$ of patients were classified as "race/ethnicity unknown" in the preimplementation cohort, compared with $4 \%$ of patients in the postimplementation period. 


\section{Process Measures}

Figure 1 shows trends in $\mathrm{OU}$ and HOT use by winter season. The percentage of patients discharged from the $\mathrm{OU}$ increased immediately after OU-HOT protocol implementation (absolute $26.9 \%$ immediate increase; $95 \% \mathrm{Cl}, 21.9-42.2$ ). The change in the proportion of OU use per season also increased (change in slope $+3.9 \%$ per season; $95 \% \mathrm{Cl}, 3.4 \%-4.4 \%)$. The percentage of patients discharged with HOT increased immediately after OU-HOT protocol implementation $(26.0 \%$ immediate change; $95 \% \mathrm{Cl}, 18.9 \%$ 33.1\%); however, the immediate increase in HOT discharges was coupled with a declining rate of HOT discharges per season in the postprotocol period compared with the preprotocol period (change in slope $-4.5 \%$ per season; $95 \% \mathrm{Cl}$, $-7.5 \%$ to $-1.5 \%$ ). Our chart review and EMR flag included 1,354 patients, or $19.0 \%$ of our cohort. Our EMR flag for HOT in the last two seasons of the study had a positive predictive value of 100\% (5 of 5 identified by EMR flag as receiving HOT were confirmed by chart review) and negative predictive value of $89 \%$ (31 of 35 identified by EMR flag as not receiving HOT were confirmed by chart review). The specificity of the EMR flag was 100\% (31 of 31 of those confirmed by chart review as not receiving HOT, who were correctly identified by EMR) and the sensitivity was 55\% ( 5 of 9 of those confirmed by chart review as receiving HOT, who were correctly identified by EMR).
TABLE. Patient Demographic and Clinical Characteristics, Preimplementation (2007-2010) and Postimplementation (2010-2019) of the OU-HOT Protocol

\begin{tabular}{|c|c|c|c|}
\hline & $\begin{array}{l}\text { Preimplementation } \\
\qquad(\mathrm{N}=2,061)\end{array}$ & $\begin{array}{l}\text { Postimplementation } \\
(\mathrm{N}=5,055)\end{array}$ & $P$ value \\
\hline Female, No. (\%) & $919(45)$ & $2,183(43)$ & .28 \\
\hline Age, mean (SD) & $10.2(5.9)$ & $10.4(5.9)$ & .30 \\
\hline Race, No. (\%) & & & $<.01$ \\
\hline White/Caucasian & $1,469(71)$ & $3,824(76)$ & \\
\hline Black/African American & $51(2)$ & $148(3)$ & \\
\hline Asian & $34(2)$ & $106(2)$ & \\
\hline Pacific Islander & $131(6)$ & $441(9)$ & \\
\hline American Indian & $23(1)$ & $75(1)$ & \\
\hline Other & $353(17)$ & $461(9)$ & \\
\hline Ethnicity, №. (\%) & & & $<.01$ \\
\hline Hispanic & $433(21)$ & $1,395(28)$ & \\
\hline Non-Hispanic & $1,017(49)$ & $3,475(69)$ & \\
\hline Unknown & $611(30)$ & $185(4)$ & \\
\hline Insurance type, No. (\%) & & & .28 \\
\hline Private & $1,002(49)$ & $2,401(48)$ & \\
\hline Government & $989(48)$ & $2,509(49)$ & \\
\hline Self/uninsured & $70(3)$ & $145(3)$ & \\
\hline PICU stay, No. (\%) & $141(7)$ & $1,207(24)$ & $<.01$ \\
\hline Mechanical ventilation, №. (\%) & $23(1)$ & $148(4)$ & $<.01$ \\
\hline Complex chronic condition, No. (\%) & $243(9)$ & $596(13)$ & $<.01$ \\
\hline
\end{tabular}

Abbreviation: PICU, pediatric intensive care unit.

\section{Primary and Secondary Outcomes}

Trends in length of stay across winter seasons are presented in Figure 2. The OU-HOT protocol was associated with an immedi- ate reduction of 30.6 hours in mean length of stay $(95 \% \mathrm{Cl},-37.1$ to -24.2). The rate of change in length of stay postimplementation did not differ significantly from the rate of change preimple-
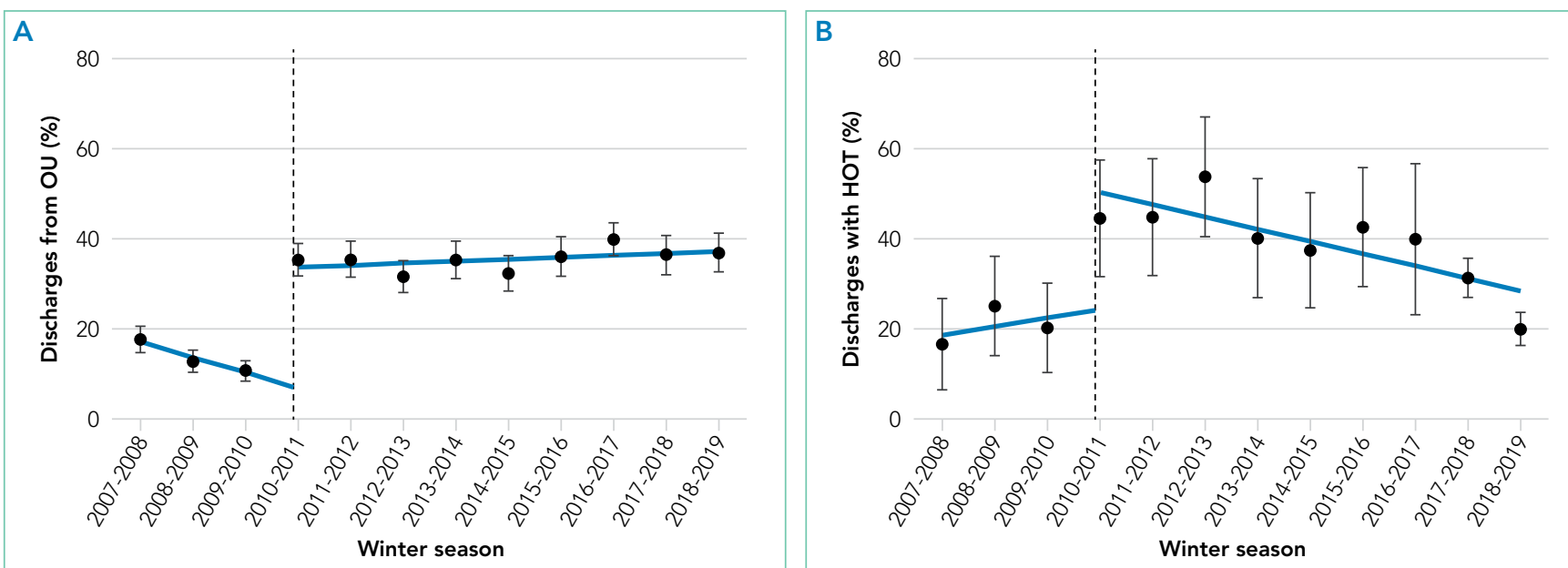

FIG 1. Process Measures, 2007-2019. Trends were analyzed via an interrupted time-series approach, with the timing of OU-HOT protocol implementation (beginning with the 2010-2011 season) marked by a dotted interruption line. Error bars denote $95 \%$ confidence intervals. (A) Percentage of patients discharged from the OU. (B) Percentage of patients discharged on HOT. 

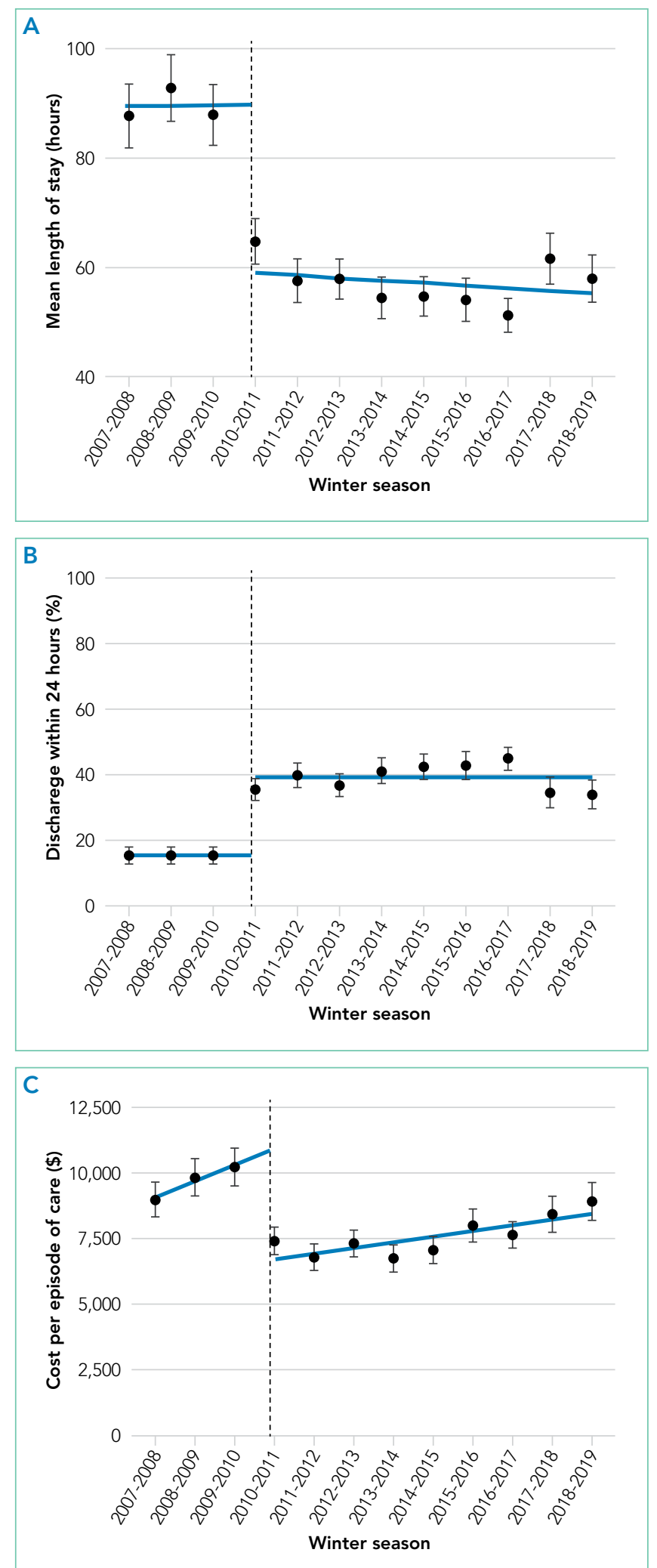

FIG 2. Primary and Secondary Outcome Measures, 2007-2019. Trends were analyzed via an interrupted time-series approach, with the timing of observation unit/home oxygen therapy (OU-HOT) protocol implementation (beginning with the 2010-2011 season) marked by a dotted interruption line. Error bars denote $95 \%$ confidence intervals. (A) Mean hospital length of stay. (B) Percentage of patients who were discharged within 24 hours. (C) Mean cost per episode of care (adjusted to 2019 dollars). mentation (change in slope -0.6 hours per season; $95 \% \mathrm{Cl},-2.3$ to 1.1 hours). The percentage of patients discharged within 24 hours of admission rose immediately after protocol implementation, by 23.8 absolute percentage points (95\% Cl, 11.7-28.8). Slopes of the preintervention and postintervention regression lines did not differ significantly (change in slope $-0.1 \%$ per season; $95 \% \mathrm{Cl},-1.4 \%$ to $1.1 \%)$. Immediate decreases in length of stay were accompanied by an immediate decrease in mean cost per episode of care $(-\$ 4,181 ; 95 \% \mathrm{Cl},-\$ 4,829$ to $-\$ 3,533)$. Protocol implementation also was associated with a decreased slope in cost postimplementation (change in slope - $\$ 403$ per season; $95 \% \mathrm{Cl},-\$ 543$ to $-\$ 264)$. The total cost savings, estimated by the product of the average cost savings per episode of care and the number of bronchiolitis admissions included in the study after OU-HOT implementation, amounted to $\$ 21.1$ million over the 9-year period, or $\$ 2.3$ million per winter season.

\section{Balancing Measures}

We observed an immediate reduction in 7-day hospital revisits $(-1.1 \%$ immediate change; $95 \% \mathrm{Cl},-1.8 \%$ to $-0.4 \%)$, but an increasing slope in revisits after implementation (change in slope $0.4 \%$ per season; $95 \% \mathrm{Cl}, 0.1 \%-0.8 \%$ ) (Figure 3 ). Stratifying revisits into ED visits and readmissions revealed that the revisit findings reflected changes in ED return visits, for which there was an immediate reduction at the time of implementation $(-1.0 \%$ immediate change; $95 \% \mathrm{Cl},-1.6 \%$ to $-0.4 \%$ ), but an increasing slope postimplementation (change in slope $0.5 \%$ per season; $95 \% \mathrm{Cl}, 0.2-0.8)$. Neither an immediate intervention effect $(0.0 \%$ immediate change; $95 \% \mathrm{Cl},-0.5 \%$ to $0.4 \%$ ) nor a change in slope (change in slope $0.0 \%$ per season; $95 \% \mathrm{Cl},-0.1 \%$ to $0.1 \%$ ) were observed for inpatient readmissions alone. The annual rate of bronchiolitis admissions to Primary Children's Hospital per 10,000 children who reside in Utah decreased after implementation of the OU-HOT protocol (immediate intervention effect -6.2 admissions; $95 \% \mathrm{Cl},-10.8$ to -1.6 ; change in slope -1.8 admissions per season; $95 \% \mathrm{Cl},-2.8$ to -0.69 ).

\section{DISCUSSION}

Our OU-HOT protocol was associated with immediate improvements in care delivered to children hospitalized for bronchiolitis, including decreased length of stay and cost savings. These improvements in outcomes largely have been sustained over a 9-year period. The OU-HOT protocol also appears to be safe as evidenced by a stable rate of readmissions over the study period and only a small increase in revisits to EDs across Intermountain Healthcare facilities, which see most children in the catchment area. Our OU-HOT protocol represents a combination of two interventions: (1) the creation of an $\mathrm{OU}$ focused on discharge within 24 to 48 hours of admission and (2) encouragement to discharge children with HOT. We found that use of the $\mathrm{OU}$ and a commitment to timely discharges has been sustained in recent years, while the commitment to HOT has appeared to wane.

Earlier investigations have evaluated the efficacy of HOT in the ED setting to prevent hospital admissions, finding high levels of caregiver comfort, estimating $\$ 1,300$ per patient cost 

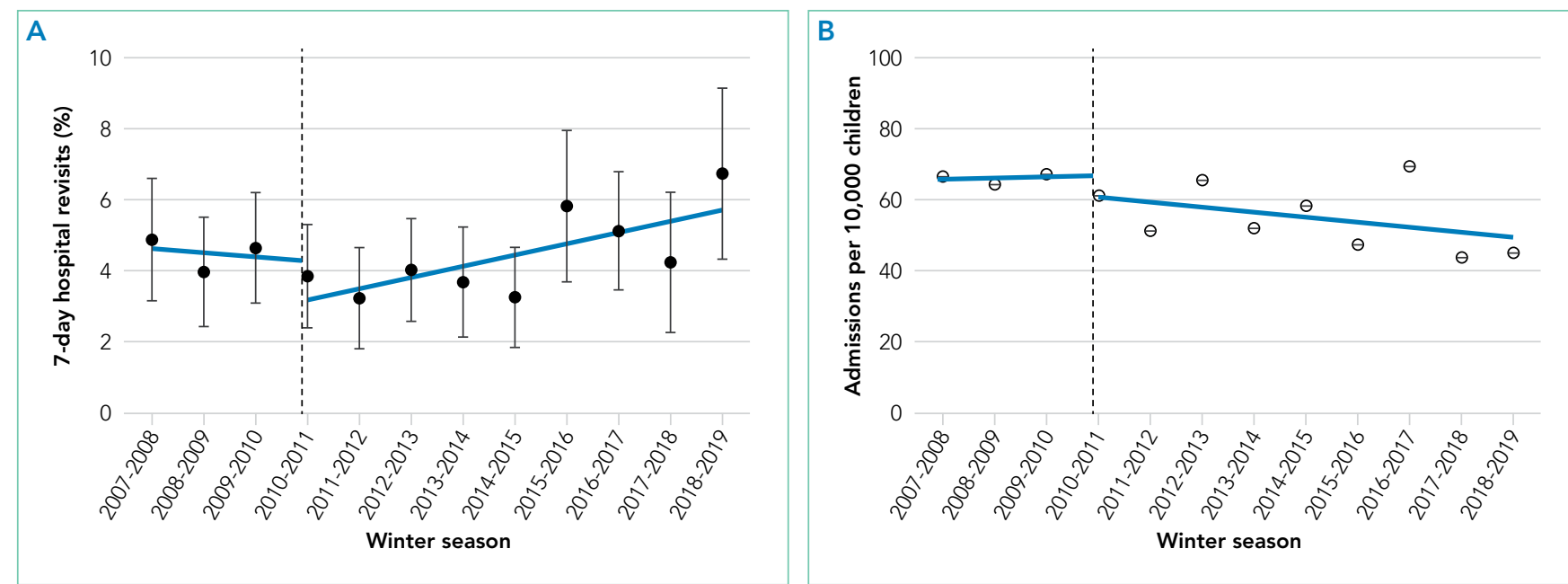

FIG 3. Balancing Measures, 2007-2019. Trends were analyzed via an interrupted time-series approach, with the timing of observation unit and home oxygen therapy (OU-HOT) protocol implementation (beginning with the 2010-2011 season) marked by a dotted interruption line. Error bars denote $95 \%$ confidence intervals. (A) Percentage of patients revisiting a hospital in the Intermountain Healthcare system within 7 days of initial discharge. (B) Annual rate of bronchiolitis admissions per 10,000 children residing in Utah.

savings, and reporting readmission rates of approximately $5 \% .^{16,23-25}$ Our study is unique in addressing HOT among a population of patients already hospitalized with bronchiolitis. The cost reductions we observed with our OU-HOT protocol were similar to those noted in the ED-based HOT protocols. However, we recorded lower readmission rates, likely because of the additional time allotted to caregivers to better gauge illness trajectory in the inpatient setting vs the ED, as well as additional time for hospitalized patients to reach the plateau or convalescent phase of illness. The small increase in ED revisits that we measured in recent years might be related to the concurrent rise in patient acuity and complexity.

Considering that length of stay has remained low despite less commitment to HOT, our results suggest that the $\mathrm{OU}$ might be the more impactful of the two interventions, and these data support the use of such a unit for a subset of patients with bronchiolitis. However, it is important to note that while the EMR HOT flag demonstrated high specificity, positive predictive value, and negative predictive value, the sensitivity was low (56\%). As a result, it is possible that we have underestimated HOT use in the 2017-2018 and 2018-2019 seasons, the final two years of the study. Alternatively, the discrepancy between sustained outcomes and lagging use of HOT could be explained by improved identification of patients who would experience the greatest benefit with oxygen in terms of length of stay reductions, with fewer patients discharged on HOT but greater per-patient benefit. Finally, in an era that encourages reduced monitor use and less aggressive response to transient mild desaturations, ${ }^{13,26,27}$ it is possible that fewer patients are identified with clinically actionable hypoxemia around the time they would be otherwise discharged.

Our OU-HOT model is not unprecedented. Increasingly, other formerly inpatient indications are being successfully managed in the observation, outpatient, and home setting, such as parenteral antibiotic treatment ${ }^{28,29}$ and chemotherapy administration. ${ }^{30}$ Considering the inpatient burden of bron- chiolitis, similar strategies to expedite discharge are needed. Although outpatient intravenous antibiotic and chemotherapy administration have been widely adopted, we are aware of only one other pediatric health care system in the United States (Children's Hospital Colorado) that routinely discharges inpatients with bronchiolitis on HOT.

This study has several limitations. First, although the interrupted time-series analysis is designed to account for trends that precede an intervention and covariates that differ before and after the intervention, it is possible that important unmeasured patient factors or changes in practice patterns differed between the pre- and post-intervention cohorts. There were no major changes to the OU-HOT protocol or discharge criteria after implementation, but individual practice management of bronchiolitis during the study period likely has evolved as new evidence emerges. Second, one could postulate that the increase in discharges within 24 hours and accompanying decreases in average length of stay and cost could be achieved by hospitalizing healthier patients over time, which the presence of an OU might incentivize. To the contrary, we found that population-based bronchiolitis admission rates have declined and disease severity appears to be increased since implementation of the OU-HOT protocol. The increase in medically complex children and PICU use in our postimplementation cohort aligns with recently published data suggesting these are national trends. ${ }^{3,31}$ Third, HOT use was estimated from a sample of the cohort using a chart review and a newly available EMR flag. A low sensitivity and a small sample for the positive predictive value are limitations of the EMR flag.

Additionally, there are almost certainly unmeasured ambulatory burdens of HOT not captured by this study. ED-based protocols have estimated that patients discharged with HOT have a median of two follow-up ambulatory visits before oxygen is discontinued ${ }^{32}$; however, the ambulatory burden associated with discharge on HOT after a hospitalization and the extent to which demographic factors affect that burden is 
unknown. Furthermore, one insurance company charged $\$ 94$ for a month of HOT in 2019; paying even a portion of this charge represents a nontrivial financial burden for many families, even considering inpatient cost savings. Although the decision to discharge on oxygen or remain hospitalized until the child did not need oxygen was left to the parents, their posthospitalization perspectives were not assessed in this study. Although reports indicate that families largely feel positive about HOT after discharge from an ED setting, with $90 \%$ of caregivers preferring HOT use to inpatient admission and most reporting no difficulty with home management, ${ }^{23}$ it is uncertain whether this would also apply after inpatient hospitalization.

\section{CONCLUSION}

The OU-HOT bronchiolitis protocol was associated with decreases in inpatient length of stay and cost while appearing safe to implement. The sustained use of the OU combined

\section{References}

1. Hasegawa K, Tsugawa Y, Brown DFM, Mansbach JM, Camargo CA. Trends in bronchiolitis hospitalizations in the United States, 2000-2009. Pediatrics. 2013;132(1):28-36. https://doi.org/10.1542/peds.2012-3877

2. Carroll KN, Gebretsadik T, Griffin MR, et al. Increasing burden and risk factors for bronchiolitis-related medical visits in infants enrolled in a state health care insurance plan. Pediatrics. 2008;122(1):58-64. https://doi.org/10.1542/peds.2007-2087

3. Fujiogi $\mathrm{M}$, Goto $\mathrm{T}$, Yasunaga $\mathrm{H}$, et al. Trends in bronchiolitis hospitalizations in the United States: 2000-2016. Pediatrics. 2019;144(6):e20192614. https:// doi.org/10.1542/peds.2019-2614

4. Schroeder AR, Mansbach JM. Recent evidence on the management of bronchiolitis. Curr Opin Pediatr. 2014;26(3):328-333. https://doi.org/10.1097 /MOP.0000000000000090

5. American Academy of Pediatrics Subcommittee on Diagnosis and Management of Bronchiolitis. Diagnosis and management of bronchiolitis. Pediatrics. 2006;118(4):1774-1793. https://doi.org/10.1542/peds.2006-2223

6. Ralston SL, Lieberthal AS, Meissner HC, et al; American Academy of Pediatrics. Clinical practice guideline: the diagnosis, management, and prevention of bronchiolitis. Pediatrics. 2014;134(5):e1474. https://doi.org/10.1542/peds.2014-2742

7. Riese J, Porter T, Fierce J, Riese A, Richardson T, Alverson BK. Clinical outcomes of bronchiolitis after implementation of a general ward high flow nasal cannula guideline. Hosp Pediatr. 2017;7(4):197-203. https://doi.org/10.1542 /hpeds.2016-0195

8. Perlstein $\mathrm{PH}, \mathrm{KotagalUR}, \mathrm{Bolling} \mathrm{C}$, etal. Evaluation ofanevidence-basedguidelineforbronchiolitis. Pediatrics.1999;104(6):1334-1341.https://doi.org/10.1542 /peds.104.6.1334

9. Perlstein PH, Kotagal UR, Schoettker PJ, et al. Sustaining the implementation of an evidence-based guideline for bronchiolitis. Arch Pediatr Adolesc Med. 2000;154(10):1001-1007. https://doi.org/10.1001/archpedi.154.10.1001

10. Wilson SD, Dahl BB, Wells RD. An evidence-based clinical pathway for bronchiolitis safely reduces antibiotic overuse. Am J Med Qual. 2002;17(5): 195-199. https://doi.org/10.1177/106286060201700507

11. BarbenJ,KuehniCE,TrachselD,HammerJ;SwissPaediatricRespiratory Research Group. Management of acute bronchiolitis: can evidence based guidelines alter clinical practice? Thorax. 2008;63(12):1103-1109. https://doi.org/10.1136 thx.2007.094706

12. Bryan MA, Desai AD, Wilson L, Wright DR, Mangione-Smith R. Association of bronchiolitis clinical pathway adherence with length of stay and costs Pediatrics. 2017;139(3):e20163432. https://doi.org/10.1542/peds.2016-3432

13. Mittal S, Marlowe L, Blakeslee S, et al. Successful use of quality improvement methodology to reduce inpatient length of stay in bronchiolitis through judicious use of intermittent pulse oximetry. Hosp Pediatr. 2019;9(2):73-78. https://doi.org/10.1542/hpeds.2018-0023

14. Macias CG, Mansbach JM, Fisher ES, et al. Variability in inpatient management of children hospitalized with bronchiolitis. Acad Pediatr. 2015;15(1): 69-76. https://doi.org/10.1016/j.acap.2014.07.005

15. Mittal V, Hall M, Morse R, et al. Impact of inpatient bronchiolitis clinical practice guideline implementation on testing and treatment. J Pediatr 2014;165(3):570-6.e3. https://doi.org/10.1016/j.jpeds.2014.05.021

16. Bajaj L, Turner CG, Bothner J. A randomized trial of home oxygen therapy from the emergency department for acute bronchiolitis. Pediatrics. with declining use of HOT suggests that the OU might be the more impactful intervention. As previously inpatient indications such as parenteral antibiotics and chemotherapy increasingly have been administered in observation and outpatient settings, bronchiolitis appears ideal for a similar strategy that allows patients to spend less time in the hospital. Studies are needed to understand the outpatient burden of HOT and the generalizability of our findings.

Disclosures: Dr. Coon is the recipient of an Intermountain-Stanford Collaboration Grant (NCT03354325), which funded a randomized controlled trial for patients hospitalized with bronchiolitis.

Funding: This investigation was supported by the University of Utah Population Health Research (PHR) Foundation, with funding in part from the National Center for Research Resources and the National Center for Advancing Translational Sciences, National Institutes of Health, through Grant 5UL1TR001067-05 (formerly 8UL1TR000105 and UL1RR025764).

2006;117(3):633-640. https://doi.org/10.1542/peds.2005-1322

17. Sandweiss DR, Mundorff MB, Hill T, et al. Decreasing hospital length of stay for bronchiolitis by using an observation unit and home oxygen therapy. JAMA Pediatr. 2013;167(5):422-428. https://doi.org/10.1001/jamapediatrics.2013.1435

18. National Bureau of Economic Research. ICD-9-CM to and from ICD-10-CM and ICD-10-PCS crosswalk or general equivalence mappings. Accessed December 2, 2020. http://www.nber.org/data/icd9-icd-10-cm-and-pcs-crosswalk-general-equivalence-mapping.html

19. Utah Department of Health, Indicator-Based Information System for Public Health. Accessed February 15, 2020. https://ibis.health.utah.gov/ibisph-view

20. James BC, Savitz LA. How Intermountain trimmed health care costs through robust quality improvement efforts. Health Aff (Millwood). 2011;30(6):11851191. https://doi.org/10.1377/hlthaff.2011.0358

21. Penfold RB, Zhang F. Use of interrupted time series analysis in evaluating health care quality improvements. Acad Pediatr. 2013;13(6 Suppl):S38-44. https://doi.org/10.1016/j.acap.2013.08.002

22. StataCorp. Stata Statistical Software: Release 15. StataCorp LLC; 2017.

23. Freeman JF, Deakyne S, Bajaj L. Emergency department-initiated home oxygen for bronchiolitis: a prospective study of community follow-up, caregiver satisfaction, and outcomes. Acad Emerg Med. 2017;24(8):920-929. https:// doi.org/10.1111/acem.13179

24. Freeman JF, Brou L, Mistry R. Feasibility and capacity for widespread use of emergency department-based home oxygen for bronchiolitis. Am J Emerg Med. 2017;35(9):1379-1381. https://doi.org/10.1016/j.ajem.2017.03.069

25. Halstead S, Roosevelt G, Deakyne S, Bajaj L. Discharged on supplemental oxygen from an emergency department in patients with bronchiolitis. Pediatrics. 2012;129(3):e605-610. https://doi.org/10.1542/peds.2011-0889

26. Quinonez RA, Coon ER, Schroeder AR, Moyer VA. When technology creates uncertainty: pulse oximetry and overdiagnosis of hypoxaemia in bronchiolitis. BMJ. 2017;358:j3850. https://doi.org/10.1136/bmj.j3850

27. Burrows J, Berg K, McCulloh R. Intermittent pulse oximetry use and length of stay in bronchiolitis: bystander or primary Driver? Hosp Pediatr. 2019;9(2): 142-143. https://doi.org/10.1542/hpeds.2018-0183

28. Norris AH, Shrestha NK, Allison GM, et al. 2018 Infectious Diseases Society of America clinical practice guideline for the managementofoutpatientparenteral antimicrobial therapy. Clin InfectDis. 2019;68(1):e1-e35. https://doi.org/10.1093 /cid/ciy745

29. Williams DN, Baker CA, Kind AC, Sannes MR. The history and evolution of outpatient parenteral antibiotic therapy (OPAT). Int J Antimicrob Agents. 2015;46(3):307-312. https://doi.org/10.1016/j.ijantimicag.2015.07.001

30. Beaty RS, Bernhardt MB, Berger AH, Hesselgrave JE, Russell HV, Okcu MF. Inpatient versus outpatient vincristine, dactinomycin, and cyclophosphamide for pediatric cancers: quality and cost implications. Pediatr Blood Cancer. 2015;62(11):1925-1928. https://doi.org/10.1002/pbc.25610

31. Coon ER, Stoddard G, Brady PW. Intensive care unit utilization after adoption of a ward-based high-flow nasal cannula protocol. J Hosp Med. 2020;15(6):325-330. https://doi.org/10.12788/jhm.3417

32. Freeman JF, Weng H-YC, Sandweiss D. Outpatient management of home oxygen for bronchiolitis. Clin Pediatr (Phila). 2015;54(1):62-66. https://doi. org/10.1177/0009922814547564 